\title{
Preparation and Microwave Absorbing Characteristics of Multi-Walled Carbon Nanotube/Chiral-Polyaniline Composites
}

\author{
Jiaoqiang Zhang*, Changming Shi, Tiezheng Ji, Guanglei Wu, Kaichang Kou \\ Key Laboratory of Space Applied Physics and Chemistry of Ministry of Education, Department of Applied \\ Chemistry, School of Science, Northwestern Polytechnical University, Xi'an, China \\ Email: zhangjq@nwpu.edu.cn
}

Received 17 June 2014; revised 15 July 2014; accepted 21 July 2014

Copyright (C) 2014 by authors and Scientific Research Publishing Inc.

This work is licensed under the Creative Commons Attribution International License (CC BY).

http://creativecommons.org/licenses/by/4.0/

(c) (i) Open Access

\section{Abstract}

The multi-walled carbon nanotubes (MWCNTs)/chiral-polyaniline composite was synthesized by in-situ chemical polymerization. Morphology, structure as well as thermal stability of the hybrid composites were characterized by using various techniques. Moreover, the complex permeability, permittivity, and microwave absorbing characteristics of the MWCNTs/chiral-polyaniline composites have been studied. Compared with those of the polyaniline (PANI) and MWCNTs, the real part $\left(\varepsilon_{r}^{\prime}\right)$ and imaginary part $\left(\varepsilon_{r}^{\prime \prime}\right)$ of the complex permittivity as well as dielectric dissipation factor of the MWCNTs/chiral-PANI composites were much greater, while the real part $\left(\mu_{r}^{\prime}\right)$ and imaginary part $\left(\mu_{r}^{\prime \prime}\right)$ of the complex permeability and the magnetic dissipation factor were smaller. The results indicate that the microwave absorption of MWCNTs/chiral-PANI composites was mainly attributed to the dielectric loss rather than magnetic loss.

\section{Keywords}

Conducting Polymers, Composites, Dielectric Properties, Properties and Characterization, Thermogravimetric Analysis (TGA)

\section{Introduction}

In recent years, electromagnetic interference (EMI) shielding problems are increasing in electronic and military ${ }^{*}$ Corresponding author.

How to cite this paper: Zhang, J.Q., Shi, C.M., Ji, T.Z., Wu, G.L. and Kou, K.C. (2014) Preparation and Microwave Absorbing Characteristics of Multi-Walled Carbon Nanotube/Chiral-Polyaniline Composites. Open Journal of Polymer Chemistry, 4, 62-72. http://dx.doi.org/10.4236/ojpchem.2014.43008 
communication owing to sensitive electronic devices and densely packed systems [1]. A variety of research works have been carried out to investigate the dielectric property, EMI shielding and microwave-absorption [2]-[9] for protecting the workspace, environment and sensitive circuits against microwave radiation emitting from computers and telecommunication equipments [10]. The absorption and the interference shielding of electromagnetic wave have been very important issues for commercial and military purposes. And with the development of radar, the stealth technique becomes the most typical application of electromagnetic wave absorption technology. By reducing the detectability of aircrafts or warships, of which the radar cross-section (RCS) is a measure, they could evade radar detection, which affects not only the mission success rate but also survival of them in the hostile territory.

Microwave absorbers are able to absorb the incident radiation in synchronized frequencies. There are many kinds of electromagnetic absorbers. Compared to conventional metal-based microwave absorbers, conductive polymer composites have gained popularity recently due to their light weight, flexibility, versatility, resistance to corrosion, and low-cost industrial processing [11]-[14]. Generally, electromagnetic wave absorption characteristic of a material depends on many factors, including its dielectric properties, magnetic properties, thickness, frequency range, aspect ratio and the filler's intrinsic conductivity [1] [11] [15].

Nano-materials have received increasingly more attention for their potential applications as practical structural and functional materials. Since the discovery in 1991 [16], carbon nanotubes (CNTs) have attracted more and more interest for their distinguished properties and promising future applications. The unique mechanical properties of CNTs such as their high conductivity, small diameter, the enormous aspect ratio, and super mechanical strength and stiffness make them a potential structural element for creating conductive composites for highperformance EMI shielding materials at low filling concentration [17]. Further potential advantages, such as their electrical and thermal conductivity together with a low density [18]-[23], make CNTs as ultimate fillers for polymers. MWCNTs exhibit excellent mechanical, thermal, and electrical properties, etc. [24]. Recently, many researchers studied the MWCNTs composites containing different polymer matrix, such as polystyrene (PS) [10], epoxy [4], poly (methylmethacrylate) (PMMA) [25], PANI [10], polypyrrole (PPY) [10], PU [4] [26] [27], poly (vinyl alcohol) [28], etc., and also explored their potential applications.

PANI is one of the most important conducting polymers [29] [30] because of its low cost of its monomer, its easy preparation, its higher conductivity, its unique electrical, optical, and optoelectrical properties and other relevant properties in such application fields as chemo- or biosensors, electromagnetic shielding, anticorrosion, electrode materials for secondary batteries, electrochromic devices, and membrane separations [31] [32]. Furthermore, PANI is unique because of its reverse acid-base doping/dedoping behavior [33] [34]. Chiral polymers discriminate between left (L) and right (R) circular polarized light in their optoelectronic properties [33]. Nowadays, chiral polymers are discussed for several applications. There is a special interest in chiral functional polymers [34]. With regard to the use of chiral materials as electromagnetic wave absorbers, Varadan et al. first reported that conductive chiral polymers showed excellent absorption properties of electromagnetic waves [35].

In this paper, we prepared MWCNTs/chiral-PANI composites using the conventional chemical oxidative polymerization of aniline(ANI) in the presence of (1S)-(+)-10- or (1R)-(-)-10-camphorsulfonic acid. An EMI shielding efficiency up to $-14.93 \mathrm{~dB}$ at the frequency of $6.7 \mathrm{GHz}$ was obtained and EMI shielding mechanism of MWCNTs/chiral-PANI composites were studied. The contribution of PANI's chirality and its doping ratio to EMI shielding efficiency were also investigated.

\section{Experimental}

\subsection{Materials}

The MWCNTs were purchased from Chengdu Organic Chemicals Co. Ltd., Chinese Academy of Sciences (COCC), they were synthesized by a chemical vapor deposition method with a carbon mass fraction of about $95 \%$ and were 0 - $8 \mathrm{~nm}$ in diameter and $10-30 \mu \mathrm{m}$ in length. ANI and concentrated ammonium hydroxide solution were purchased from Fu Chen Chemical Technology Company Ltd., Shanghai, China. ANI was distilled under vacuum prior to usage. Concentrated ammonium hydroxide was diluted with deionized water for the dedoping experiments. (1S)-(+)-10-camphorsulfonic acid (D-CSA, 99\% purity), and (1R)-(-)-10-camphorsulfonic acid (L-CSA, 99\%) were purchased from Dibo Chemical Technology Company Ltd., Shanghai, China. 


\subsection{Synthesis of MWCNTs/Chiral-PANI Composites}

\subsubsection{MWCNTs/EB Composites}

$0.2 \mathrm{~g}$ acid-treated MWCNTs was put into round-bottom flask and then added $10 \mathrm{~mL}$ deionized water, sonicated for $0.5 \mathrm{~h}$ to form uniform suspension, added $4.6 \mathrm{~g}(0.05 \mathrm{~mol})$ ANI monomer, the reaction mixture was kept in ice bath $\left(-5^{\circ} \mathrm{C}\right)$ with continuous stirring for keeping the temperature constant and stable during reaction. PANI in this form (Emeraldine base (EB)) was synthesized by oxidative chemical polymerization of ANI using ammonium persulfate (APS) at a molar ratio of 1:1. APS aqueous solution (0.05 mol APS dissolved into $50 \mathrm{~mL} 1$ $\mathrm{M} \mathrm{HCl}$ ) was added dropwise in three separate portions with half an hour interval into the above mixture under stirring. The polymerization process was carried out at $-5^{\circ} \mathrm{C}$ for $6 \mathrm{~h}$ with stirring after which it was left overnight. Finally the product was filtered and washed with deionized water and then with ethanol. The polymer was transferred to a $250 \mathrm{~mL}$ beaker containing $200 \mathrm{~mL}$ of $3 \% \mathrm{NH}_{4} \mathrm{OH}$, stirred for 12 hours, and subsequently filtered to collect the MWCNTs/EB (EBC) composite powder, and dried in a vacuum oven at $80^{\circ} \mathrm{C}$ for 8 hours and stored at room temperature.

\subsubsection{MWCNTs/L-PANI (or MWCNTs/D-PANI) Composites}

EBC composite powder, which was prepared by a synthetic method mentioned in Section 2.2.1 was added to a $250 \mathrm{~mL}$ beaker first, and then $0.06 \mathrm{~mol}$ D-CSA (or L-CSA) was added into reaction vessel. The mixture was kept at room temperature with stirring and keeping the temperature constant during reaction. The doping process was carried out for $36 \mathrm{~h}$ with continuously stirring. Finally the product was filtered and washed with deionized water and then with ethanol. The prepared MWCNTs/chiral-PANI composites was then dried in a vacuum oven at $80^{\circ} \mathrm{C}$ for 8 hours and stored at room temperature.

\subsection{Characterization of the Composites}

The Fourier transform infrared (FT-IR) spectra of MWCNTs/L-PANI (L-C), MWCNTs/D-PANI (D-C) and EBC composites were obtained using a FT-IR spectrophotometer (Perkin-Elmer, USA, model Spectrum-BX) at room temperature. X-ray diffraction (XRD) measurements using $\mathrm{Cu}$ Ka radiation were performed with a powder X-ray diffractometer (RINT 1100, Rigaku). Surface morphology of the composites was observed by transmission electron microscopy (TEM) observations (JEOL-2010, $200 \mathrm{kV}$ ) at various magnifications. The samples for TEM measurements were prepared by adding a few drops of ethanol suspension of the composites on carbon mesh-coated copper grids. Thermogravimetric analysis (TGA) was carried out by a SEIKO I \& E SSC/570 thermal controller and a TG/DTA 30 at a heating rate of $10^{\circ} \mathrm{C} / \mathrm{min}$ in $\mathrm{N}_{2}$ atmosphere in the temperature range of $0^{\circ} \mathrm{C}-800^{\circ} \mathrm{C}$. A network analyzer HP8720Et was employed to determine the values of $\varepsilon_{r}^{\prime}, \varepsilon_{r}^{\prime \prime}, \mu_{r}^{\prime}$, and $\mu_{r}^{\prime \prime}$ at different frequencies by using a reflection/transmission technique. For this, toroidal-shaped samples of $3 \mathrm{~mm}$ inner diameter and $7 \mathrm{~mm}$ outer diameter were tightly inserted into a standard coaxial line. Sample lengths approximately equal to one quarter of the guide wavelength were chosen for accurate measurements, so the sample lengths varied from 2 to $3 \mathrm{~mm}$.

\section{Results and Discussion}

\subsection{FT-IR Spectroscopy}

The FT-IR spectra of L-C, EBC and D-C are shown in Figure 1. The characteristic absorption bands at 1589 and $1499 \mathrm{~cm}^{-1}$ are attributed to $\mathrm{C}=\mathrm{C}$ stretching of the quinoid and $\mathrm{C}=\mathrm{C}$ stretching of the benzenoid rings, respectively. Furthermore, the peaks at 1310 and $1160 \mathrm{~cm}^{-1}$ are ascribed to C-N and C-N+• (polaron of PANI) stretching modes, respectively, and the band at $1101 \mathrm{~cm}^{-1}$ represents the $\mathrm{C}-\mathrm{H}$ aromatic in-plane bending. These bands are in good agreement with the spectrum of PANI when in the form of an emeraldine salt [36] [37]. In addition, the bands at 821 and $583 \mathrm{~cm}^{-1}$ are assigned to the absorption of $-\mathrm{SO}_{3} \mathrm{H}$, suggesting that the prepared composites were doped with D-CSA (or L-CSA) [38]. The peak at $1120 \mathrm{~cm}^{-1}$ is assigned to C-O stretching vibration and the peak at $1720 \mathrm{~cm}^{-1}$ is attributed to $-\mathrm{C}=\mathrm{O}$ stretching vibration in the carboxyl group. This indicates the $-\mathrm{COOH}$ group functionalization of MWCNTs [39] [40].

\subsection{XRD}

The structural characteristics of EB and composite have been studied by XRD as shown in Figure 2. The $2 \theta$ 


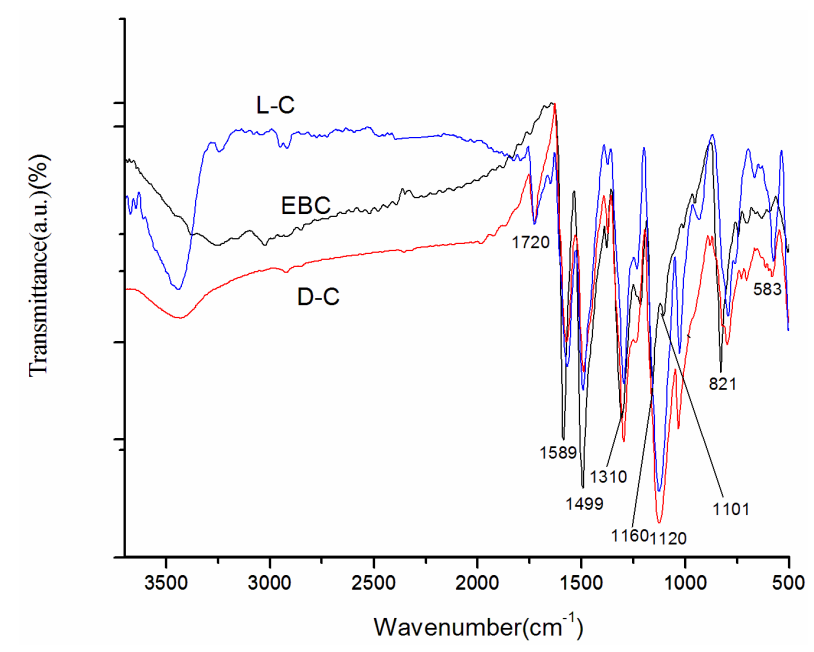

Figure 1. FT-IR spectra of EBC, L-C and D-C composites.

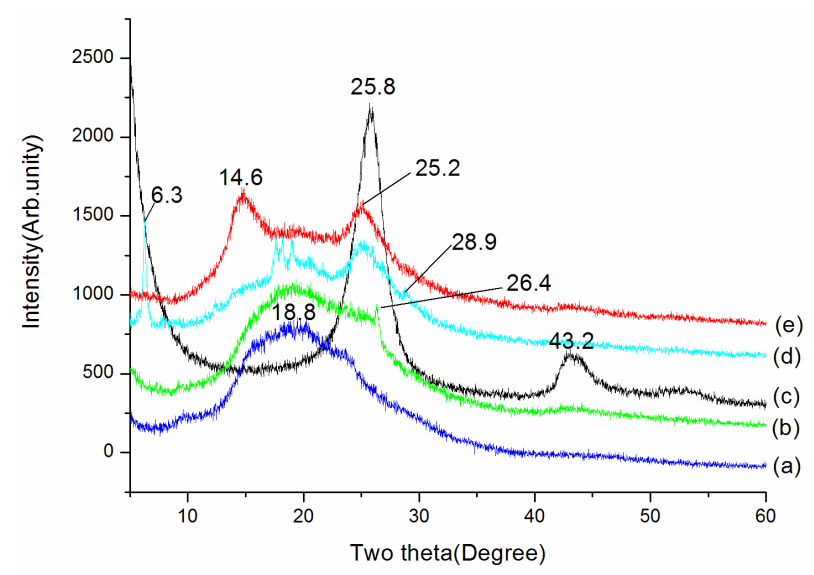

Figure 2. XRD patterns of (a) EB, (b) EBC, (c) MWCNTs, (d) L-C and (e) D-C composites.

scan shows the typical XRD pattern for EB [41]-[44] and MWCNTs [45], which are shown in Figure 2 noted with a and c, respectively. In the pattern of pristine EB, the XRD pattern exhibits a broad amorphous diffraction peak centered at $18.8^{\circ}$ indicating that the EB is fully amorphous, which may be ascribed to the periodicity parallel to the polymer chain [44]. For pristine MWCNTs, two characteristic peaks at $2 \theta=25.8^{\circ}$ and $43.2^{\circ}$ are observed (Figure 2(d)), corresponding to the intershell spacing of the concentric cylinders of graphitic carbon and small amount of catalytic particles encapsulated inside the walls of CNTs [45] [46]. For the EBC composite, the XRD data showed the characteristic EB broad peaks, besides, a small sharp peak at $2 \theta=26.4^{\circ}$ appeared. This peak can be ascribed to the peak of MWCNTs. Compared XRD patterns of L-C and D-C with MWCNTs and EB, it was clear that additional order structures have been introduced in $\mathrm{L}-\mathrm{C}$ and $\mathrm{D}-\mathrm{C}$. The spectra for the $\mathrm{D}-\mathrm{C}$ clearly reveal new diffraction peak at $2 \theta=14.6^{\circ}$, ones for $\mathrm{L}-\mathrm{C}$ peaks centered at $2 \theta=6.3^{\circ}, 17.7^{\circ}, 18.3^{\circ}$ and $19.0^{\circ}$, it can be seen that the relative intensities of MWCNTs diffraction peaks increase comparing with ones of EBC for the D-C and L-C. The appearance of the new peaks as well as the increase in peak intensity indicates an increase in the extension of microcrystalling domains in the D-C and L-C. These dominant features in D-C and L-C indicate that the formation and stability of the new structures is enhanced when chiral CSA (D- or L-) were used to dope the composites.

\subsection{TEM}

A TEM image of the MWCNTs is shown in Figure 3. The TEM image reveals that the MWCNTs are straight 


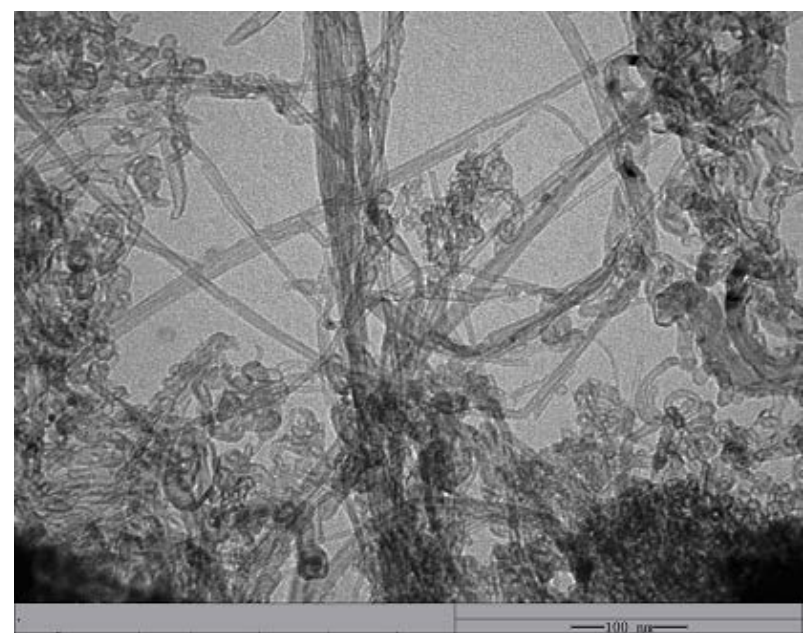

Figure 3. TEM image of MWCNTs.

with diameter $5-8 \mathrm{~nm}$, internal diameter $2-5 \mathrm{~nm}$ and length $10-30 \mu \mathrm{m}$. It can be seen in Figure 3 that MWCNTs scatter uniformity, and the surface is smooth. Figure 4(a) and Figure 4(b) show the morphologies of L-C. It can be observed that when MWCNTs coated with chiral PANI, the surface of the L-C presents some small spines like sharp needle. The length of the sharp needle is about 40 - $45 \mathrm{~nm}$, the bottom diameter of the sharp needle is about 40 - $43 \mathrm{~nm}$. Compared with the MWCNTs, it can be seen from Figure 4(b) the diameter of the L-C is about $130-140 \mathrm{~nm}$, the average thickness of coating layer is about $60 \mathrm{~nm}$ (The length of the sharp needle is not included).

\subsection{TGA}

Thermal stability testing of the composite and PANI were examined by a TGA. In Figure 5 and Figure 6 there are comparisons of the mass losses of PANI, PANI-(L-CSA), PANI-(D-CSA) and EBC composites upon heating in a nitrogen atmosphere. MWCNTs are very stable and no decomposition takes place until $600^{\circ} \mathrm{C}$ in nitrogen atmosphere [42]. PANI displays a sudden decrease in mass loss of about $34 \%$ at a temperature of $658^{\circ} \mathrm{C}$, and the onset temperature of its TGA curve is $510.5^{\circ} \mathrm{C}$, and the peak temperature is $555.5^{\circ} \mathrm{C}$ for the corresponding DTG curve, which is corresponding to the thermal decomposition reaction of PANI and the total mass loss at up to $786^{\circ} \mathrm{C}$ can be estimated to be about $40 \%$. Here, the trend of the degradation curve of EBC is similar to that of PANI, the degradation of the EBC composite is mainly controlled by that of PANI. The chiral polyanilie (PANI-(L-CSA) or PANI-(D-CSA)) shows a $26.3 \%$ mass loss from 238.93 to $375.96^{\circ} \mathrm{C}$, which is corresponding to the thermal decomposition reaction of doping CSA. After $679.85^{\circ} \mathrm{C}$, another $37.8 \%$ mass loss is observed due to the decomposition reaction of PANI, and the total mass loss at up to $786^{\circ} \mathrm{C}$ can be estimated to be about $35 \%$.

\subsection{Measurement of the Microwave Absorption Effects}

Since the absorbing properties of microwave absorbing materials can be estimated from their magnetic and dielectric properties, the essence of researching microwave absorbers is to design the compound and structure form of the materials, by adjusting and optimizing the dielectric and magnetic properties such as complex permittivity and permeability of the materials [47]. In order to investigate the intrinsic reasons for microwave absorption of the composites, we measured the complex permittivity and permeability of all the composites at the frequency range of 2 - $18 \mathrm{GHz}$. Figure 7 shows the $\varepsilon_{r}^{\prime}, \varepsilon_{r}^{\prime \prime}$ and the dielectric dissipation factor $\left(\tan \delta_{\varepsilon}=\varepsilon_{r}^{\prime \prime} /\right.$ $\varepsilon_{r}^{\prime}$ ) vs frequency spectra of the composites, CNTs, and chiral PANI. The $\varepsilon_{r}^{\prime}$ and $\varepsilon_{r}^{\prime \prime}$ of the L-PANI are 2.65 to3.14 and 0.03 to 0.25 respectively. The highest values of the $\varepsilon_{r}^{\prime}$ and $\varepsilon_{r}^{\prime \prime}$ for the D-C composite reach 9.76 and 2.76, respectively. The $\varepsilon_{r}^{\prime}, \varepsilon_{r}^{\prime \prime}$, and $\tan \delta_{\varepsilon}$ of the L-C composite range from 5.54 to 8.96 and 1.22 to 2.72 , and 0.27 to 0.36 , respectively. And those of D-C composites are 6.26 to 9.76, 1.14 to 2.76 and 0.22 to 0.31 , respectively. And those of the D-PANI are 3.11 to 4.20, 0.30 to 0.70 , and $0.09-0.21$, respectively. As can be seen, the $\varepsilon_{r}^{\prime}, \varepsilon_{r}^{\prime \prime}$, and $\tan \delta_{\varepsilon}$ increase dramatically as the addition of MWCNTs in the composites. Figure 8 shows the $\mu_{r}^{\prime}, \quad \mu_{r}^{\prime \prime}$ and magnetic dissipation factor $\left(\tan \delta_{\mu}=\varepsilon_{\mu}^{\prime \prime} / \varepsilon_{\mu}^{\prime}\right)$ vs frequency spectra of the composites, CNTs, and 


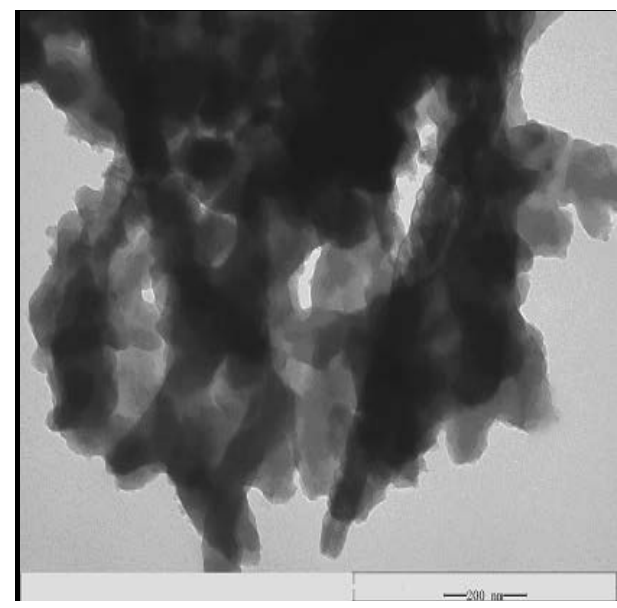

(a)

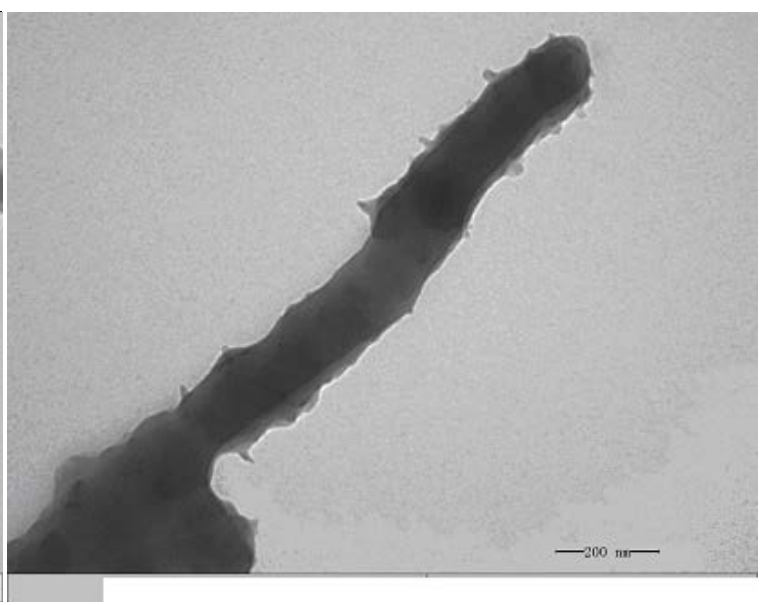

(b)

Figure 4. TEM images of L-C, (a) L-C, (b) A single L-C thread.

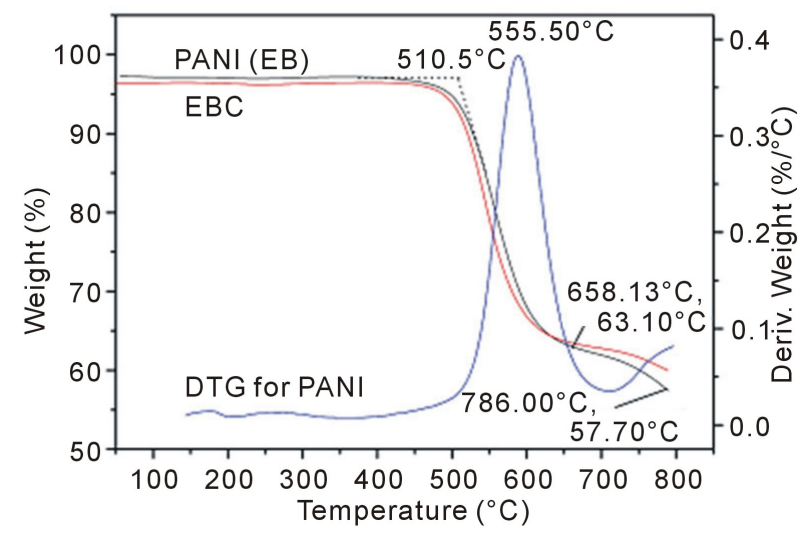

Figure 5. TGA-DTG curve of PANI and TGA curve of MWNTs/ L-PANI at a heating rate of $10^{\circ} \mathrm{C} / \mathrm{min}$ measured under a nitrogen atmosphere.

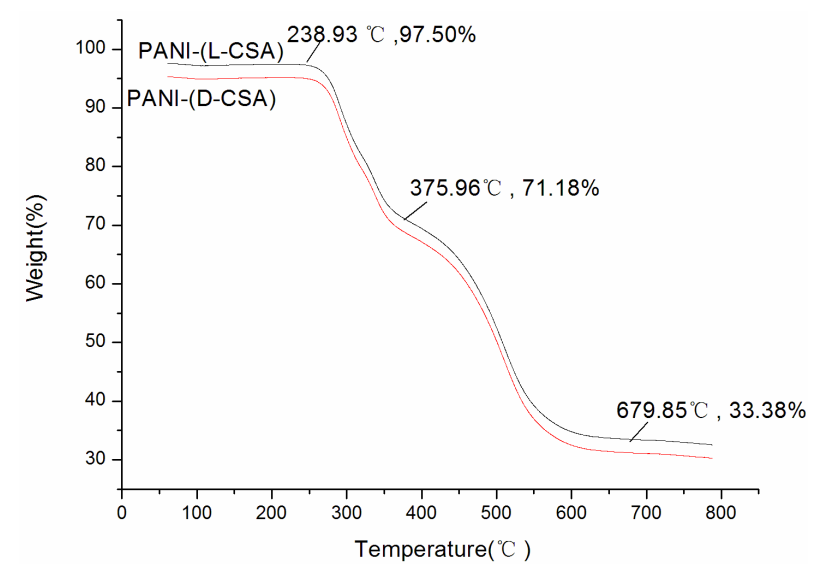

Figure 6. TGA curves of chiral PANI at a heating rate of $10^{\circ} \mathrm{C} /$ min measured under a nitrogen atmosphere.

chiral PANI. As can be seen from Figures 8(a)-(c), the $\mu_{r}^{\prime}, \mu_{r}^{\prime \prime}$, and $\tan \delta_{\mu}$ have no large change as the addition of MWCNTs in the composites. The $\mu_{r}^{\prime}, \mu_{r}^{\prime \prime}$, and $\tan \delta_{\mu}$ of the L-C composite range from 0.93 to 1.43 , 


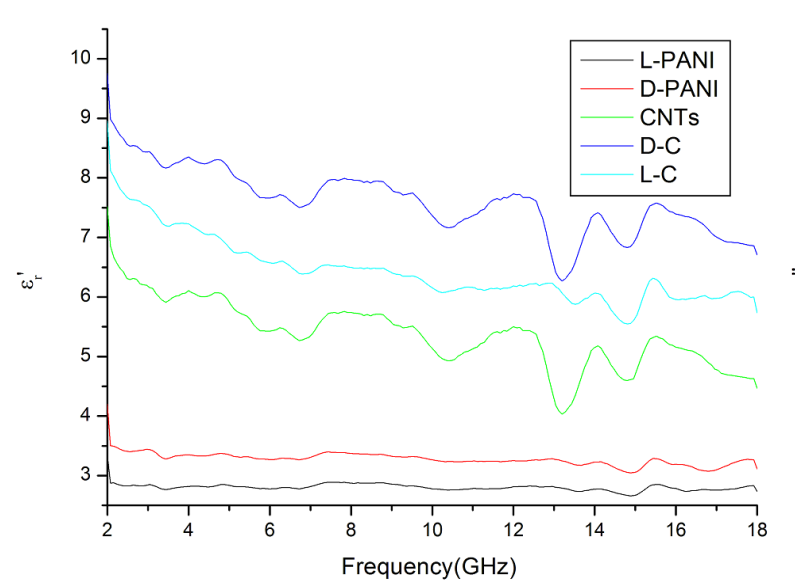

(a)

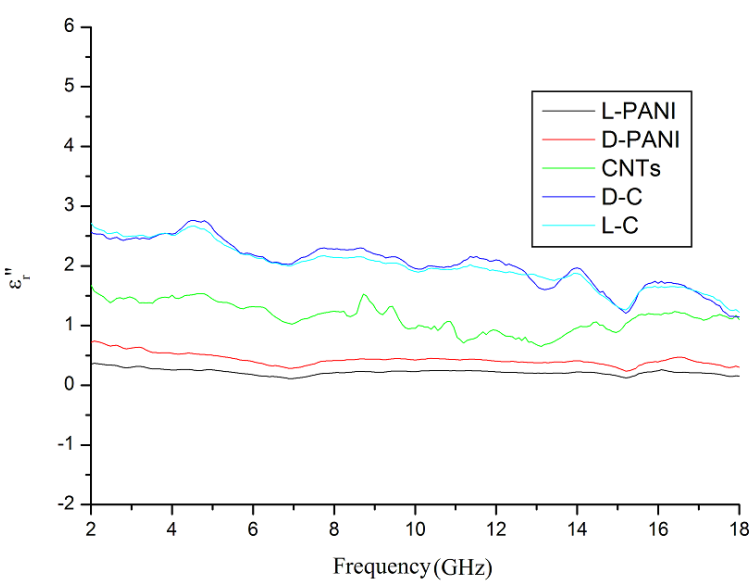

(b)

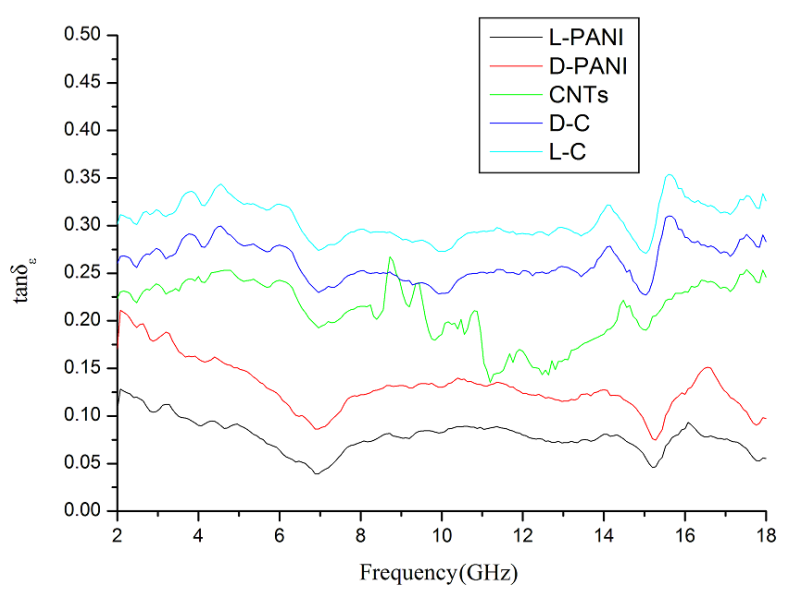

(c)

Figure 7. $\varepsilon_{r}^{\prime}, \varepsilon_{r}^{\prime \prime}$ and $\tan \delta_{\varepsilon}$ vs frequency spectra in the range of $2.0-18.0 \mathrm{GHz}$ for the composites, CNTs, and chiral PANI. (a) $\varepsilon_{r}^{\prime}$, (b) $\varepsilon_{r}^{\prime \prime}$, and (c) $\tan \delta_{\varepsilon}$.

-0.08 to 0.16 , and -0.08 to 0.15 , respectively. And those of D-C composite are 0.92 to $1.40,-0.92$ to 0.19 and -0.10 to 0.16 , respectively.

As illustrated in Figure 7 and Figure 8, it is observed that the value of dielectric loss is much higher than that of magnetic loss at the considered frequency. It indicates that the composites have a good and stable dielectric loss property. It suggests that the microwave absorption enhancement of the composites mainly results from dielectric loss, rather than magnetic loss.

To predict the microwave-absorption of all the composites, calculations were carried out to determine the reflection loss. Thus, the reflection loss R could be obtained as following [48] [49]:

The normalized input impedance $Z_{\text {in }}$ of a metal-backed microwave-absorbing layer is given by

$$
Z_{i n}=\sqrt{\frac{\mu_{r}}{\varepsilon_{r}}} \tan h\left(i \frac{2 \pi f}{c} \sqrt{\mu_{r} \varepsilon_{r}} d\right)
$$

where $\mu_{r}\left(\mu_{r}=\mu^{\prime}-i \mu^{\prime \prime}\right)$ and $\varepsilon_{r}\left(\varepsilon_{r}=\varepsilon^{\prime}-i \varepsilon^{\prime \prime}\right)$ are the relative permeability and permittivity, respectively, of the composite medium, $c$ is the velocity of electromagnetic waves in free space, $f$ is the frequency of microwaves, and $d$ is the thickness of the absorber. The reflection loss is related to $Z_{\text {in }}$ as:

$$
\text { reflection loss }(\mathrm{dB})=20 \log \frac{\left|Z_{\text {in }}-1\right|}{\left|Z_{\text {in }}+1\right|}
$$




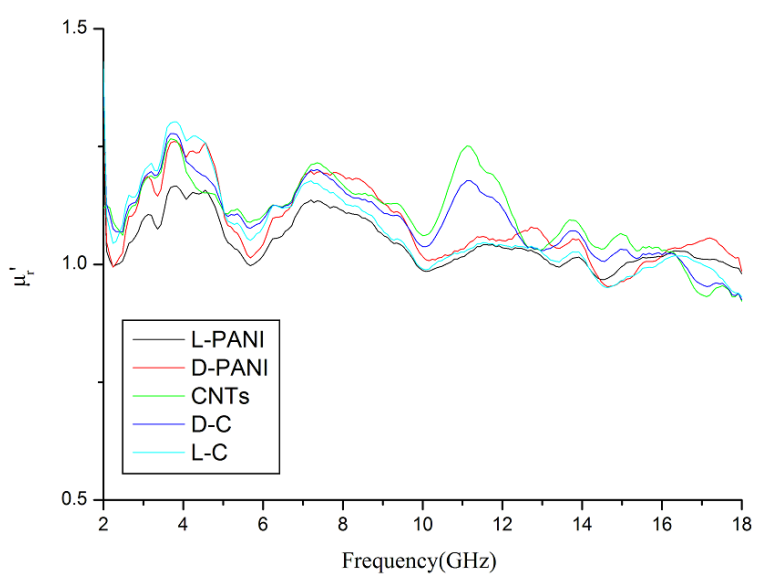

(a)

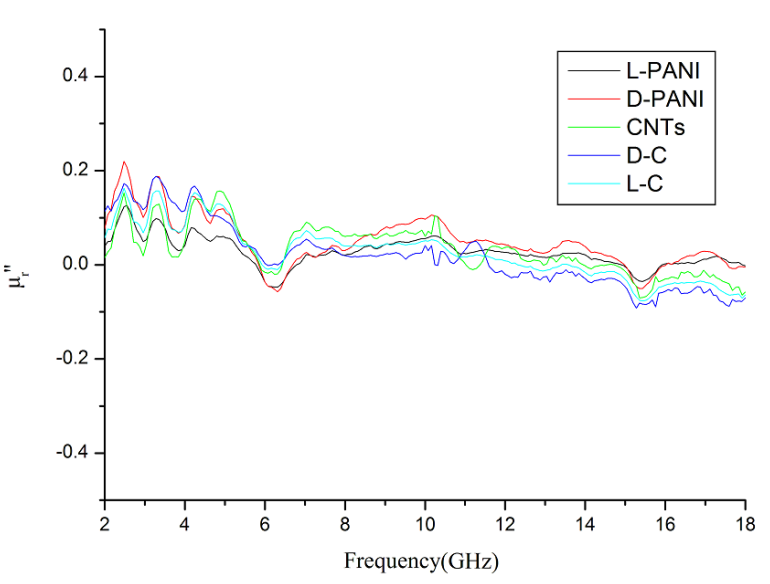

(b)

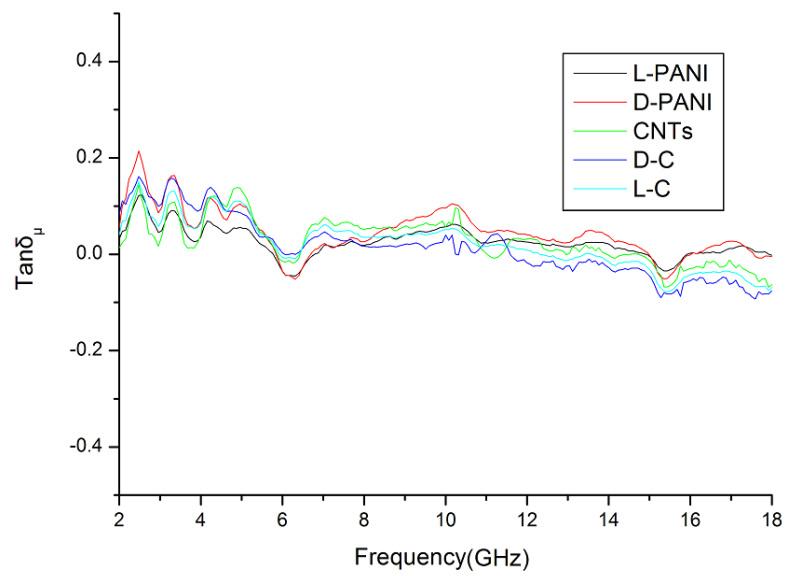

(c)

Figure 8. $\mu_{r}^{\prime}, \mu_{r}^{\prime \prime}$ and tan $\delta \mu$ vs frequency spectra in the $2.0-18.0 \mathrm{GHz}$ range for the composites, CNTs, and chiral PANI. (a) $\mu_{r}^{\prime}$, (b) $\mu_{r}^{\prime \prime}$, and (c) $\tan \delta_{\mu}$.

Thus, the surface reflectance of an absorber is a function of six characteristic parameters, viz., $f, d, \varepsilon_{r}^{\prime}, \varepsilon_{r}^{\prime \prime}$, $\mu_{r}^{\prime}$ and $\mu_{r}^{\prime \prime}$. It is possible to evaluate the numerical values of these parameters corresponding to the condition of zero reflection $\left(Z_{\text {in }}=1\right)$.

Figure 9 shows the frequency dependence of the reflection loss of the composites. It can be seen that the addition of the MWCNTs has an obvious effect on microwave absorbing properties. As seen from Figure 9, the microwave absorbing difference of the chiral PANI between L-PANI and D-PANI is visible. Although the absorption difference between L-C and D-C composite is small, it could be drawn a conclusion that the microwave absorbing property for these materials is related to their chirality. The L-C composite achieved a reflection loss below $-10 \mathrm{~dB}$ over $1.6 \mathrm{GHz}$ in the range of 5.8 to $7.4 \mathrm{GHz}$, and the maximum value was $-14.95 \mathrm{~dB}$ at $6.72 \mathrm{GHz}$, the reflection loss below $-10 \mathrm{~dB}$ for the $\mathrm{D}-\mathrm{C}$ was over $1.8 \mathrm{GHz}$ in the range of 5.2 to $7.0 \mathrm{GHz}$, and the maximum value was $-13.11 \mathrm{~dB}$ at $5.92 \mathrm{GHz}$. And the absorption curve illustrates that the maximum value of reflection loss for the MWCNTs is $-8.89 \mathrm{~dB}$ at $7.04 \mathrm{GHz}$, and that for L-PANI and D-PANI are $-1.23 \mathrm{~dB}$ at 10.32 $\mathrm{GHz}$ and $-6.34 \mathrm{~dB}$ at $10.16 \mathrm{GHz}$, respectively. When the MWCNTs were coated with PANI, the microwave absorption peaks of the corresponding composites moved to the lower frequency.

\section{Conclusion}

In conclusion, MWCNTs/chiral-PANI composites have been prepared successfully by in-situ chemical oxidative polymerization of ANI as monomer, L-CSA and D-CSA as chiral doping reagents, APS as oxidative onto 


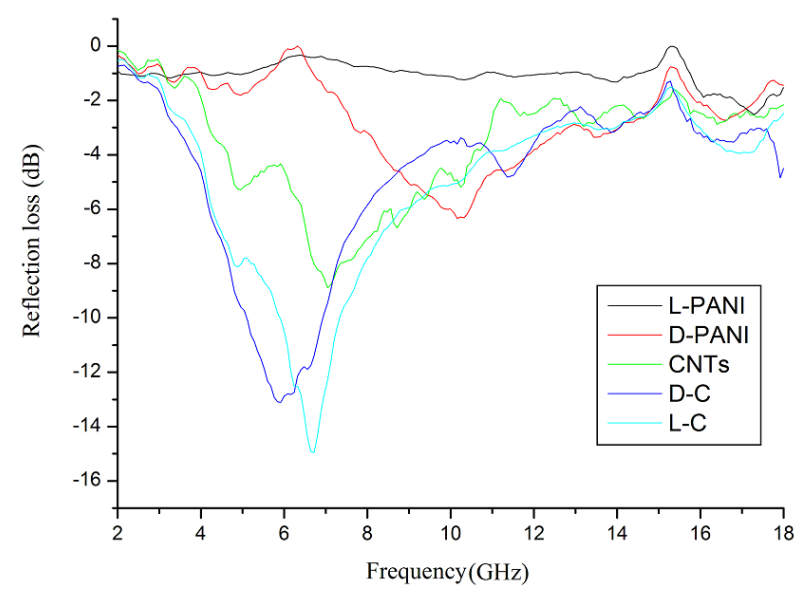

Figure 9. Calculated reflection loss for different composite specimens with maximum attenuation.

MWCNTs. The characterizations of the molecular structure indicate that additional order has been introduced in L-C and D-C composites due to the presence of chiral CSA. The surface of L-C composite presents some small PANI spines with height of about $40-45 \mathrm{~nm}$ and bottom diameter of about $40-43 \mathrm{~nm}$. Finally, the complex permeability, permittivity, and microwave absorption of the composites have been studied. Compared with those of the L-PANI and D-PANI, the $\varepsilon_{r}^{\prime}, \varepsilon_{r}^{\prime \prime}$ and $\tan \delta_{\varepsilon}$ of the L-C and D-C are much greater, while the $\mu_{r}^{\prime}, \mu_{r}^{\prime \prime}$ and $\tan \delta_{\mu}$ are smaller. It is found that the permittivity has remarkable effect on microwave absorption property of the samples, but the magnetization degree and magnetic loss of the samples are small, within the frequency range of $2.0 \sim 18.0 \mathrm{GHz}$, the D-C composite achieved a reflection loss below $-5 \mathrm{~dB}$ over $6.1 \mathrm{GHz}$ in the range of $4.1 \sim 10.2 \mathrm{GHz}$, and the maximum value was $-14.95 \mathrm{~dB}$ at $6.72 \mathrm{GHz}$, the reflection loss below $-5 \mathrm{~dB}$ for the L-C composite is over $-4.5 \mathrm{GHz}$ in the range of $4.0 \sim 8.5 \mathrm{GHz}$, and the maximum value is $-13.11 \mathrm{~dB}$ at 5.92 $\mathrm{GHz}$. It is concluded that the microwave absorbing property of the chiral composites is function of its chirality.

\section{References}

[1] Park, K.Y., Lee, S.E., Kim, C.G. and Han, J.H. (2006) Fabrication and Electromagnetic Characteristics of Electromagnetic Wave Absorbing Sandwich Structures. Composites Science and Technology, 66, 576-584. http://dx.doi.org/10.1016/j.compscitech.2005.05.034

[2] Che, R.C., Peng, L.M., Duan, X.F., Chen, Q. and Liang, X.L. (2004) Microwave Absorption Enhancement and Complex Permittivity and Permeability of Fe Encapsulated within Carbon Nanotubes. Advanced Materials, 16, 401-405. http://dx.doi.org/10.1002/adma.200306460

[3] Yang, Y.L., Gupta, M.C., Dudley, K.L. and Lawrence, R.W. (2005) Novel Carbon Nanotube-Polystyrene foam Composites for Electromagnetic Interference Shielding. Nano Letters, 5, 2131-2134. http://dx.doi.org/10.1021/nl051375r

[4] Li, N., Huang, Y., Du, F., He, X.B., Lin. X., Gao, H.J., et al. (2006) Electromagnetic Interference (EMI) Shielding of Single-Walled Carbon Nanotube Epoxy Composites. Nano Letters, 6, 1141-1145. http://dx.doi.org/10.1021/nl0602589

[5] Cao, M.S., Shi, X.L., Fang, X.Y., Jin, H.B., Hou, Z.L., Zhou, W., et al. (2007) Microwave Absorption Properties and Mechanism of Cagelike $\mathrm{ZnO} / \mathrm{SiO}_{2}$ Nanocomposites. Applied Physics Letters, 91, Article ID: 203110.

[6] Huang, Y., Li, N., Ma, Y.F., Feng, D., Li, F.F., He, X.B., et al. (2007) The Influence of Single-Walled Carbon Nanotube Structure on The Electromagnetic Interference Shielding Efficiency of Its Epoxy Composites. Carbon, 45, 16141621. http://dx.doi.org/10.1016/j.carbon.2007.04.016

[7] Shi, X.L., Cao, M.S., Yuan, J.Q., Zhao, L., Kang, Y.Q., Fang, X.Y. and Chen, Y.J. (2008) Nonlinear Resonant and High Dielectric loss Behavior of CdS/alpha- $\mathrm{Fe}_{2} \mathrm{O}_{3}$ Heterostructure Nanocomposites. Applied Physics Letters, 93, Article ID: 183118.

[8] Lakshmi, K., John, H., Mathew, K.T., Joseph, R. and George, K.E. (2009) Microwave Absorption, Reflection and EMI Shielding of PU-PANI Composite. Acta Materialia, 57, 371-375. http://dx.doi.org/10.1016/j.actamat.2008.09.018

[9] Song, W.L., Cao, M.S., Hou, Z.L., Fang, X.Y., Shi, X.L. and Yuan, J. (2009) High Dielectric Loss and Its Monotonic 
Dependence of Conducting Dominated Multiwalled Carbon Nanotubes/Silica Nanocomposite on Temperature Ranging from 373 to $873 \mathrm{~K}$ in X-Band. Applied Physics Letters, 94, Article ID: 233110.

[10] Wang, Y. and Jing, X. (2005) Intrinsically Conducting Polymers for Electromagnetic Interference Shielding. Polymers for Advanced Technologies, 16, 344-351. http://dx.doi.org/10.1002/pat.589

[11] Joo, J. and Lee, C.Y. (2000) High Frequency Electromagnetic Interference Shielding Response of Mixtures and Multilayer Films Based on Conducting Polymers. Journal of Applied Physics, 88, 513-518. http://dx.doi.org/10.1063/1.373688

[12] Xiang, C.S., Pan, Y.B., Liu, X.J., Sun, X.W., Shi, X.M. and Guo, J.K. (2005) Microwave Attenuation of Multiwalled Carbon Nanotube-Fused Silica Composites. Applied Physics Letters, 87, Article ID: 123103.

[13] Yang, Y.L., Gupta, M.C., Dudley, K.L. and Lawrence, R.W. (2005) Conductive Carbon Nanofiber-Polymer Foam Structures. Advanced Materials, 17, 1999-2003.

[14] Bryning, M.B., Islam, M.F., Kikkawa, J.M. and Yodh, A.G. (2005) Very Low Conductivity Threshold in Bulk Isotropic Single-Walled Carbon Nanotube-Epoxy Composites. Advanced Materials, 17, 1186-1191. http://dx.doi.org/10.1002/adma.200401649

[15] Chung, D.D.L. (2001) Electromagnetic Interference Shielding Effectiveness of Carbon Materials. Carbon, 39, $279-285$. http://dx.doi.org/10.1016/S0008-6223(00)00184-6

[16] Iijima, S. (1991) Helical Microtubules of Graphitic Carbon. Nature, 354, 56-58. http://dx.doi.org/10.1038/354056a0

[17] Liu, Z.F., Bai, G., Huang, Y., Ma, Y.F., Du, F., Li, F.F., Guo, T.Y. and Chen, Y.S. (2007) Reflection and Absorption Contributions to The Electromagnetic Interference Shielding of Single-Walled Carbon Nanotube/Polyurethane Composites. Carbon, 45, 821-827. http://dx.doi.org/10.1016/j.carbon.2006.11.020

[18] Ajayan, P.M., Stephan, O., Colliex, C. and Trauth, D. (1994) Aligned Carbon Nanotube Arrays Formed by Butting A Polymer Resin-Nanotube Composite. Science, 265, 1212-1214. http://dx.doi.org/10.1126/science.265.5176.1212

[19] Treacy, M.M.J., Ebbesen, T.W. and Gibson, J.M. (1996) Exceptionally High Young's Modulus Observed for Individual Carbon Nanotubes. Nature, 381, 678-680. http://dx.doi.org/10.1038/381678a0

[20] Ebbesen, T.W., Lezec, H.J., Hiura, H., Benett, J.W., Ghaemi, H.F. and Thio, T. (1996) Electrical Conductivity of Individual Carbon Nanotubes. Nature, 382, 54-56. http://dx.doi.org/10.1038/382054a0

[21] Lu, J.P. (1997) Elastic Properties of Carbon Nanotubes and Nanoropes. Physical Review Letters, 79, 1297-1300. http://dx.doi.org/10.1103/PhysRevLett.79.1297

[22] Wong, E.W., Sheehan, P.E. and Lieber, C.M. (1997) Nanobeam Mechanics: Elasticity, Strength and Toughness of Nanorods and Nanotubes. Science, 277, 1971-1975. http://dx.doi.org/10.1126/science.277.5334.1971

[23] Gojny, F.H. and Schulte, K. (2004) Functionalisation Effect on The Thermomechanical Behaviour of Multi-Wall Carbon Nanotube/Epoxy-Composites. Composites Science and Technology, 64, 2303-2308. http://dx.doi.org/10.1016/j.compscitech.2004.01.024

[24] Schartel, B., Potschke, P., Knoll, U. and Abdel-Goad, M. (2005) Fire Behavior of Polyamide/Multiwall Carbon Nanotube Nanocomposites. European Polymer Journal, 41, 1061-1070. http://dx.doi.org/10.1016/j.eurpolymj.2004.11.023

[25] Kim, H.M., Kim, K., Lee, S.J., Joo, J., Yoon, H.S., Cho, S.J., Lyu, S.C. and Lee, C.J. (2004) Charge Transport Properties of Composites of Multiwalled Carbon Nanotube with Metal Catalyst and Polymer: Application to Electromagnetic Interference Shielding. Current Applied Physics, 4, 577-580. http://dx.doi.org/10.1016/j.cap.2004.01.022

[26] Ma, C.C.M., Huang, Y.L., Kuan, H.C. and Chiu, Y.S. (2005) Preparation and Electromagnetic Interference Shielding Characteristics of Novel Carbon-Nanotube/Siloxane/Poly-(Urea Urethane) Nanocomposites. Journal of Polymer Science Part B: Polymer Physics, 43, 345-358. http://dx.doi.org/10.1002/polb.20330

[27] Wu, H.L., Ma, C.C.M., Yang, Y.T., Kuan, H.C., Yang, C.C. and Chiang, C.L. (2006) Morphology, Electrical Resistance, Electromagnetic Interference Shielding and Mechanical Properties of Functionalized MWNT and Poly(Urea Urethane) Nanocomposites. Journal of Polymer Science Part B: Polymer Physics, 44, 1096-1105. http://dx.doi.org/10.1002/polb.20766

[28] Liu, L., Barber, A.H., Nuriel, S. and Wagner, H.D. (2005) Mechanical Properties of Functionalized Single-Walled Carbon-Nanotube/Poly(Vinyl Alcohol) Nanocomposites. Advanced Functional Materials, 15, 975-980. http://dx.doi.org/10.1002/adfm.200400525

[29] MacDiarmid, A.G., Chiang, J.C., Richter, A.F. and Epstein, A.J. (1987) Polyaniline: A New Concept in Conducting Polymers. Synthetic Metals, 18, 285-290. http://dx.doi.org/10.1016/0379-6779(87)90893-9

[30] Cao, Y., Smith, P. and Heeger, A.J. (1992) Counter-Ion Induced Processibility of Conducting Polyaniline and of Conducting Polyblends of Polyaniline in Bulk Polymers. Synthetic Metals, 48, 91-97. http://dx.doi.org/10.1016/0379-6779(92)90053-L

[31] Cao, Y., Andreatta, A., Heeger, A.J. and Smith, P. (1989) Influence of Chemical Polymerization Conditions on the 
Properties of Polyaniline. Polymer, 30, 2305-2311. http://dx.doi.org/10.1016/0032-3861(89)90266-8

[32] MacDiarmid, A.G. and Epstein, A.J. (1995) Secondary Doping in Polyaniline. Synthetic Metals, 69, 85-92. http://dx.doi.org/10.1016/0379-6779(94)02374-8

[33] Ding, S.J., Zhang, C.L., Yang, M., Qu, X.Z., Lu, Y.F. and Yang, Z.Z. (2006) Template Synthesis of Composite Hollow Spheres Using Sulfonated Polystyrene Hollow Spheres. Polymer, 47, 8360-8366. http://dx.doi.org/10.1016/j.polymer.2006.10.001

[34] Huang, J.X. and Kaner, R.B. (2004) A General Chemical Route to Polyaniline Nanofibers. Journal of the American Chemical Society, 126, 851-855. http://dx.doi.org/10.1021/ja0371754

[35] Varadan, V.V., Lakhtakia, A. and Varadan, V.K. (1988) Equivalent Dipole Moments of Helical Arrangements of Small, Isotropic, Point-Polarizable Scatters: Application to Chiral Polymer Design. Journal of Applied Physics, 63, 280-284. http://dx.doi.org/10.1063/1.340289

[36] Peng, X.Y., Luan, F., Liu, X.X., Diamond, D. and Lau, K.T. (2009) pH-Controlled Morphological Structure of Polyaniline during Electrochemical Deposition. Electrochimica Acta, 54, 6172-6177. http://dx.doi.org/10.1016/j.electacta.2009.05.075

[37] Xing, S.X., Zheng, H.W. and Zhao, G.K. (2008) Preparation of Polyaniline Nanofibers via a Novel Interfacial Polymerization Method. Synthetic Metals, 158, 59-63. http://dx.doi.org/10.1016/j.synthmet.2007.12.004

[38] Zhang, L.J. and Wan, M.X. (2002) Synthesis and Characterization of Self-Assembled Polyaniline Nanotubes Doped with D-10-Camphorsulfonic Acid. Nanotechnology, 13, 750-755. http://dx.doi.org/10.1088/0957-4484/13/6/311

[39] Dhand, C., Arya, S.K., Singh, S.P., Singh, B.P., Datta, M. and Malhotra, B.D. (2008) Preparation of Polyaniline/Multiwalled Carbon Nanotube Composite by Novel Electrophoretic Route. Carbon, 46, 1727-1735. http://dx.doi.org/10.1016/j.carbon.2008.07.028

[40] Yan, X.B., Han, Z.J., Yang, Y. and Tay, B.K. (2007) Fabrication of Carbon Nanotube-Polyaniline Composites via Electrostatic Adsorption in Aqueous Colloids. The Journal of Physical Chemistry C, 111, 4125-4131. http://dx.doi.org/10.1021/jp0651844

[41] Feng, W., Bai, X.D., Lian, Y.Q., Liang, J., Wang, X.G. and Yoshino, K. (2003) Well-Aligned Polyaniline/CarbonNanotube Composite Films Grown by in-Situ Aniline Polymerization. Carbon, 41, 1551-1557. http://dx.doi.org/10.1016/S0008-6223(03)00078-2

[42] Karim, M.R., Lee, C.J., Park, Y.T. and Lee, M.S. (2005) SWNTs Coated by Conducting Polyaniline: Synthesis and Modified Properties. Synthetic Metals, 151, 131-135. http://dx.doi.org/10.1016/j.synthmet.2005.03.012

[43] Luzny, W. and Banka, E. (2000) Relations between the Structure and Electric Conductivity of Polyaniline Protonated with Camphorsulfonic Acid. Macromolecules, 33, 425-429. http://dx.doi.org/10.1021/ma9913663

[44] Moon, Y.B., Cao, Y., Smith, P. and Heeger, A.J. (1989) X-Ray Scattering from Crystalline Polyaniline. Polymer Communications, 30, 196-199.

[45] Maser, W.K., Benito, A.M., Callejas, M.A., Seeger, T., Martínez, M.T., Schreiber, J., Muszynski, J., Chauvet, O., Osváth, Z., Koós, A.A. and Biró, L.P. (2003) Synthesis and Characterization of New Polyaniline/Nanotube Composites. Materials Science and Engineering: C-Materials for Biological Applications, 23, 87-91. http://dx.doi.org/10.1016/S0928-4931(02)00235-7

[46] Liu, S.W., Yue, J. and Wehmschulte, R.J. (2002) Large Thick Flattened Carbon Nanotubes. Nano Letters, 2, 14391442. http://dx.doi.org/10.1021/nl0257869

[47] Zhao, D.L., Li, X. and Shen, Z.M. (2008) Microwave Absorbing Property and Complex Permittivity and Permeability of Epoxy Composites Containing Ni-Coated and Ag Filled Carbon Nanotubes. Composites Science and Technology, 68, 2902-2908. http://dx.doi.org/10.1016/j.compscitech.2007.10.006

[48] Kim, S.S., Jo, S.B., Gueon, K.I., Choi, K.K., Kim, J.M. and Chum, K.S. (1991) Complex Permeability and Permittivity and Microwave Absorption of Ferrite-Rubber Composite in X-Band Frequencies. IEEE Transactions on Magnetics, 27, 5462-5464. http://dx.doi.org/10.1109/20.278872

[49] Singh, P., Babbar, V.K., Razdan, A., Puri, R.K. and Goel, T.C. (2000) Complex Permittivity, Permeability, and X-Band Microwave Absorption of CaCoTi Ferrite Composites. Journal of Applied Physics, 87, 4362-4366. http://dx.doi.org/10.1063/1.373079 
Scientific Research Publishing (SCIRP) is one of the largest Open Access journal publishers. It is currently publishing more than 200 open access, online, peer-reviewed journals covering a wide range of academic disciplines. SCIRP serves the worldwide academic communities and contributes to the progress and application of science with its publication.

Other selected journals from SCIRP are listed as below. Submit your manuscript to us via either submit@scirp.org or Online Submission Portal.
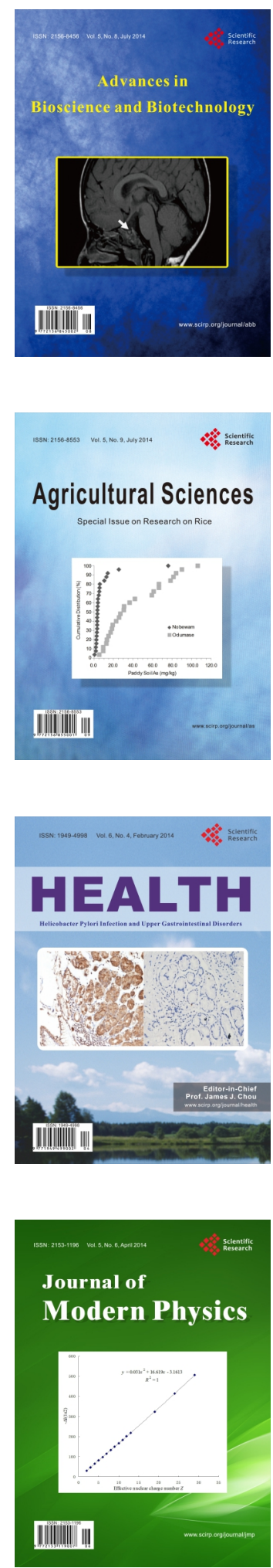
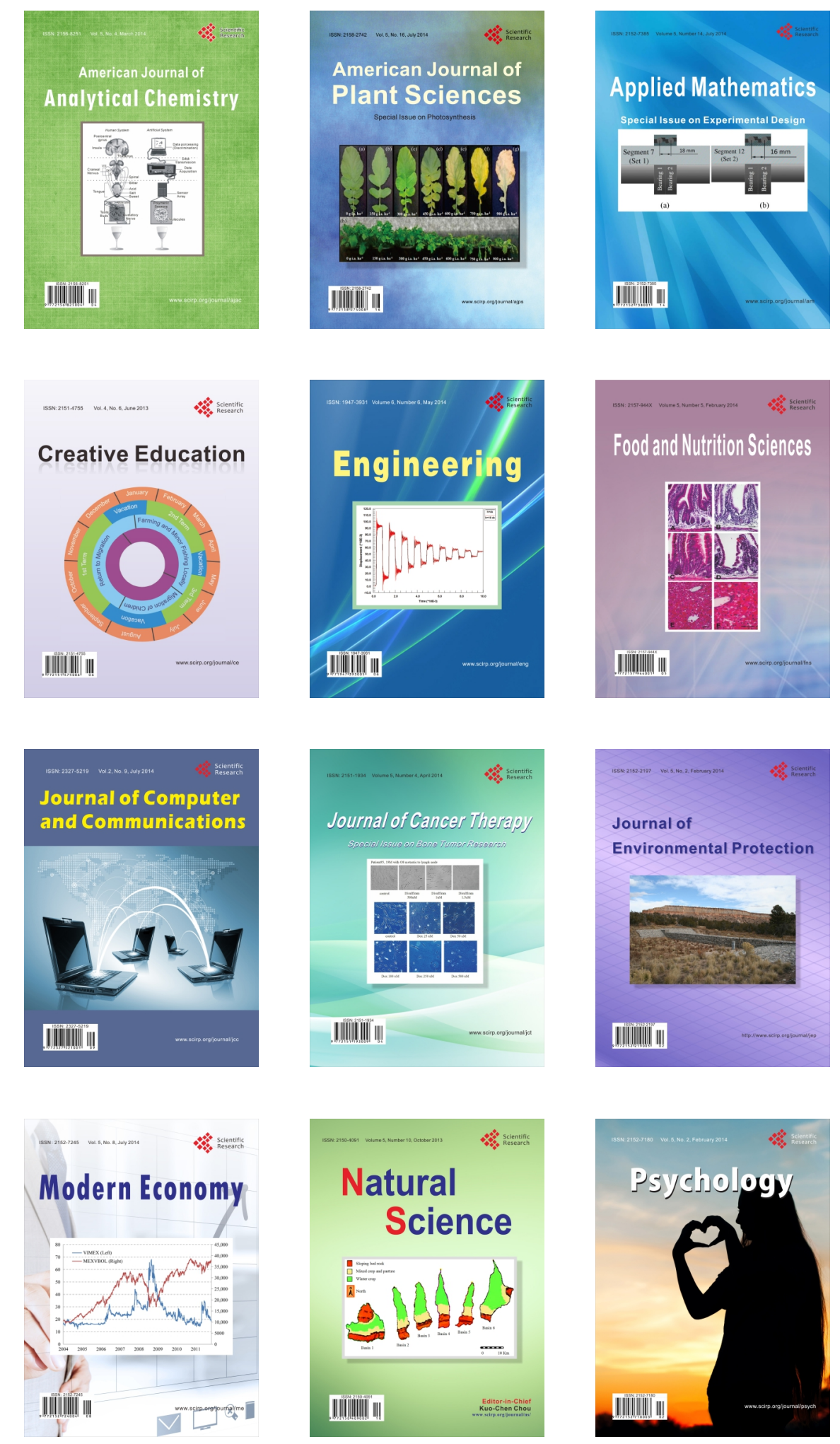